\title{
Mise au point de jouguets monobovins pour la culture attelée dans la zone de savane du Nord Cameroun
}

\author{
E. Vall ${ }^{1}$ O. Abakar ${ }^{2}$ E. Kpoumié ${ }^{3}$
}

\section{Mots-clés}

Bovin - Harnachement - Culture attelée - Energie animale - Cameroun.

\begin{abstract}
Résumé
Au Nord Cameroun, les agriculteurs utilisent une paire de bœufs ou bien un âne pour le labour et les travaux d'entretien des cultures. Avant 1997, I'usage du monobœuf pour la culture attelée n'était pas pris en compte par le développement. Or sur une parcelle cultivée, le passage d'une paire de bovins dans l'interligne pose des difficultés au conducteur (piétinement des plants, casse des tiges par la chaîne de traction, difficultés de manœuvre en bout de rangs). Le joug "enjambeur » n'a pas été adopté par les paysans car son envergure $(160 \mathrm{~cm})$ rend l'attelage difficile à manœuvrer en bout de ligne. L'attelage monobovin permet de résoudre ces difficultés. En 1998, à la demande de la Société de développement du coton du Cameroun (Sodécoton), I'Institut de recherche agricole pour le développement (Irad) a mis au point et testé en milieu paysan deux modèles de jouguets monobovins. Le modèle Irad BF se caractérise par une pièce en bois en demi-cercle qui épouse la forme de la bosse du zébu (7 500 Fcfa). Le modèle Irad BT se compose de trois pièces de bois ajustées en triangle (8 $700 \mathrm{Fcfa}$ ). Une série de 50 exemplaires de chaque modèle ont été testés par des agriculteurs volontaires. Une majorité a déclaré préférer le modèle Irad BF car il était parfaitement stable et mieux ajusté sur la bosse de l'animal. Les points faibles du modèle Irad BT ont été les suivants : un prix trop élevé, un défaut d'assemblage des montants, une instabilité dans les virages. Depuis 1999, la Sodécoton a vendu 620 exemplaires du modèle Irad BF. Le travail se poursuit par la mise au point $d^{\prime}$ une charrette monobovine.
\end{abstract}

\section{INTRODUCTION}

Au Nord Cameroun (figure 1), les paysans utilisent généralement une paire de bovins pour réaliser les travaux de labour, de sarclage et de buttage (9). Pour le labour, le paysan emploie un joug de garrot « court » (entre axe $80 \mathrm{~cm}$ ). Le joug de sarclage « enjambeur» (entre axe $160 \mathrm{~cm}$ ) proposé par le développement n'a pas été

1. Cirad-emvt, TA30/A, Campus international de Baillarguet, 34398 Montpellier Cedex 5, France

2. Irad, Station polyvalente, BP 415, Garoua, Cameroun

3. Etudiant Fasa, Université de Dschang, BP 222, Dschang, Cameroun adopté par les paysans car, en raison de son envergure, l'attelage est trop difficile à conduire lorsqu'il faut effectuer un demi-tour en fin de ligne. Généralement, pour le sarclage et le buttage les paysans attèlent une paire de bovins au joug court. Cependant, le passage d'un tel attelage dans des rangs de culture pose beaucoup de difficultés au conducteur (piétinement des cultures, balayage oblique de la chaîne de traction et casse des plants, difficultés de manœuvre en bout de rangs), surtout lorsque les plants de coton, de maïs ou encore de sorgho atteignent plusieurs décimètres. Les paysans qui utilisent la traction mono-asine ou bien mono-équine ne rencontrent pas ces difficultés. Dans la partie soudanienne du bassin cotonnier (pluviométrie : > $1000 \mathrm{~mm}$ ), les paysans sont demandeurs d'attelages puissants en raison de la pression des adventices et d'animaux suffisamment résistants aux maladies 


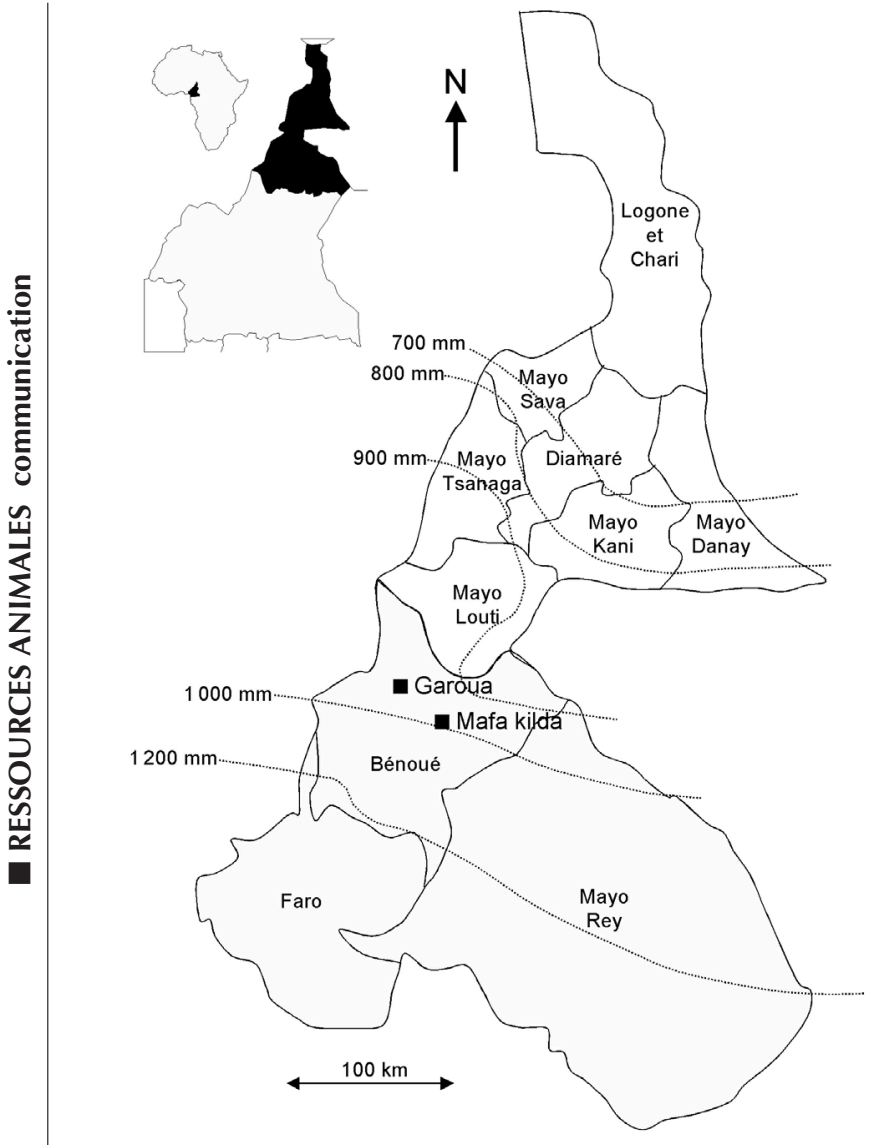

Figure 1 : Nord Cameroun administratif et points d'essai des jouguets (village de Mafa Kilda et départements du Mayo Rey, Bénoué et Faro).

parasitaires, deux conditions qui, réunies, disqualifient les équidés pour envisager leur utilisation à grande échelle. Partant de ce constat, l'Institut de la recherche agricole pour le développement (Irad) et la Société de développement du coton au Cameroun (Sodécoton) ont décidé de mettre au point un harnachement pour attelage monobovin, visant en priorité la clientèle des agriculteurs du sud du bassin cotonnier (départements du Mayo Rey, Bénoué et Faro).

La Sodécoton s'est intéressée au thème du monobovin dès 1997. Un premier prototype de jouguet avait été testé sans succès dans le Mayo Rey : il n'était pas stable sur l'animal et se renversait dans les virages. En 1998, la Sodécoton a demandé à l'Irad de mettre au point un jouguet monobovin. Elle souhaitait qu'il soit faisable localement, d'un prix inférieur à $10000 \mathrm{Fcfa}$, adaptable sur la grande majorité des gabarits des bovins de la région, absolument stable pour ne pas se renverser dans les demi-tours, d'une envergure inférieure à $70 \mathrm{~cm}$ pour passer entre les interlignes, résistant à une élévation subite de l'effort de traction en cas de blocage sur une souche et, enfin, convenablement dimensionné et ajusté pour ne pas blesser l'animal. Plusieurs modèles devaient être conçus pour offrir un choix aux paysans selon leurs possibilités et leurs préférences.

\section{MATERIEL ET METHODES}

Le travail a été conduit en deux étapes : i) la mise au point des prototypes en milieu contrôlé en suivant le cahier des charges fixé par la Sodécoton ; ii) la réalisation de tests de vulgarisation (appelés « prévulgarisation » dans la suite du texte) en milieu paysan en collaboration avec le commanditaire de l'étude.

\section{Etape 1 : prototypes}

Les prototypes ont été conçus et testés en station au cours du premier trimestre de 1998. Ce matériel étant destiné à des zébus, deux premiers principes de construction ont été retenus. Premièrement, un harnais rigide a été préféré au harnais souple, ce dernier étant moins adapté à l'anatomie du bovin (8). Deuxièmement, le joug de tête a été délaissé au profit du joug de garrot car, sur le zébu, ce dernier est plus efficace et plus économique $(5,8)$. Ces premiers principes établis, l'étude a commencé à partir d'expériences antérieures qui n'avaient pas abouti : le jouguet Atécam (4), le jouguet en demi-cercle testé par le Centre d'étude rurale de Ngong (Cern) et le prototype Sodécoton 1997. Ainsi, deux autres principes de construction ont été retenus : i) un jouguet de forme triangulaire de type « chinois » (2); ii) un jouguet en demi-cercle épousant la forme de la bosse du zébu. Le bois (madriers et chevrons) et le fer à béton, facilement disponibles localement, ont été les principaux matériaux retenus pour la fabrication. Pour les traits, il a été nécessaire, en accord avec la Sodécoton, d'importer des sangles de Nylon de très haute résistance car les matériaux disponibles localement n'étaient pas satisfaisants (trop fragiles, putrescibles, etc.).

Cette étape a permis de déterminer les dimensions caractéristiques des jouguets et d'estimer les prix de revient. Les auteurs ont pris soin d'ajuster les deux modèles sur des zébus Bororo akou de gabarit moyen (350 à $450 \mathrm{~kg}$ de poids vif), car ces animaux sont les plus utilisés pour la culture attelée au Nord Cameroun (6). Avant d'aboutir aux deux modèles de jouguet « prévulgarisables », de nombreux ajustements ont été nécessaires par essai ou erreur sur les premiers prototypes. Ces ajustements ont porté principalement sur les points suivants : la partie avant au contact du garrot, la dimension des pièces (épaisseur, diamètre, etc.), les techniques d'assemblage et la longueur des traits. Ainsi, à chaque étape de la conception, les auteurs ont procédé à un essayage sur plusieurs zébus de tailles différentes. Les détails de cette étape ont été volontairement omis de la suite du texte pour ne faire état que du résultat obtenu, c'est-à-dire deux modèles de jouguet prêts à être testés par les agriculteurs.

\section{Etape 2 : prévulgarisation}

$\mathrm{Au}$ terme de cette étape, une première série de 100 jouguets, 50 Irad BT et 50 Irad BF, ont été fabriqués et prévulgarisés dans les régions cotonnières de Garoua Est et de Touboro. Au cours de la saison des pluies de 1998, l'Irad a effectué six démonstrations et les agents de la Sodécoton une soixantaine. A l'issue de chaque démonstration, des exemplaires des deux jouguets étaient laissés aux groupes d'agriculteurs volontaires pour la durée de la saison des cultures. Finalement, une enquête a été menée pour recueillir les impressions et suggestions d'une vingtaine de groupes d'agriculteurs et d'individus (paysans et agents d'encadrement) ayant participé à cette campagne de prévulgarisation.

Parallèlement, un test comparatif des deux jouguets a été effectué dans un village proche de Garoua, Mafa Kilda. En plus des avis des agriculteurs, ont été relevés les performances et les comportements des attelages sur des modes de cultures aussi différents que le coton et le maïs (3).

Les conclusions de l'étude ont été rendues à la Sodécoton fin 1998 (10). L'année suivante, la Sodécoton a mis en vente ce type de matériel et le propose dorénavant dans son catalogue. Elle s'occupe de la fabrication dans ses propres ateliers.

\section{RESULTATS}

Des jouguets de garrot prenant appui sur la bosse du zébu ont ainsi été mis au point. Ils sont maintenus en place par une cravate 
passant sous le fanon du bovin. Au travail (figure 2), on constate que le jouguet se place en position oblique selon la ligne de traction. Les parties en contact avec la bosse sont aplanies et arrondies sur les arêtes de manière à maximiser la surface tractante et éviter les blessures. Les bras du jouguet font office de balancier et le stabilisent dans la position de travail. La cravate est maintenue serrée sans excès par un système de double anneaux. Le mode d'emploi du jouguet est indiqué dans l'encadré 1 .

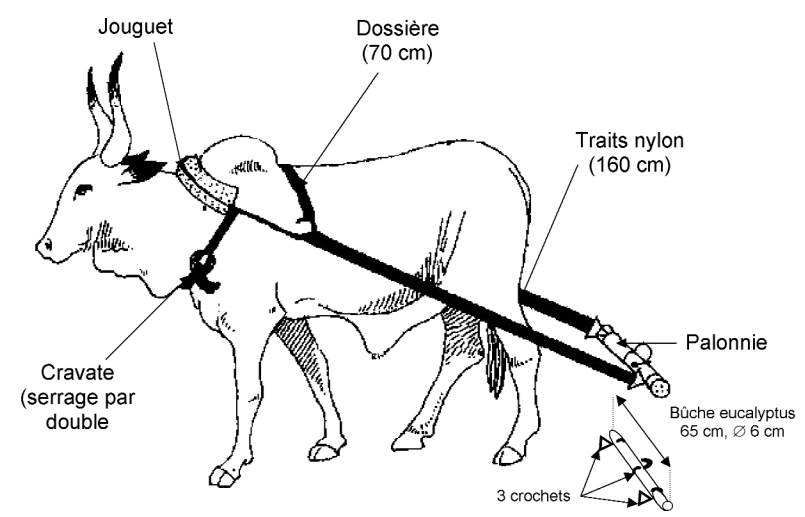

Figure 2 : jouguet monobovin et accessoires.

\section{Les deux prototypes de jouguets}

Deux modèles de jouguets monobovins ont été proposés aux agriculteurs. Ils se distinguent par des principes de fabrication différents de la partie antérieure, en contact avec la bosse.

\section{Jouguet Irad BT}

Ce jouguet (figure 3 ) est inspiré du modèle Atécam réalisé par Le Thiec à Garoua en 1994 (4). Il est construit en bois d'eucalyptus * . Il a une forme de triangle (d'où son nom, BT = bois triangle). Les montants latéraux (6 à $8 \mathrm{~cm}$ de diamètre) sont fixés par un boulon (Trcc $10 \mathrm{x} 120)$. La pièce centrale $(9 \mathrm{~cm}$ de diamètre) qui prend appui sur la bosse est pincée entre les deux montants et emprisonnée par un système d'encoches. Des lanières de caoutchouc renforcent le maintien de cette pièce. Les traits sont arrimés au jouguet dans deux encoches taillées aux extrémités des bras. La cravate se compose de deux brins de sangle en Nylon (70 et $110 \mathrm{~cm}$ ). Le serrage de la cravate se fait au moyen d'un système de double anneaux $(6 \mathrm{~cm}$ de diamètre). La position de la cravate sur les montants latéraux est maintenue par deux encoches circulaires. Compte tenu des nombreuses découpes de bois (encoches et angles), mais aussi de l'irrégularité des fûts d'eucalyptus, ce modèle est compliqué à réaliser.

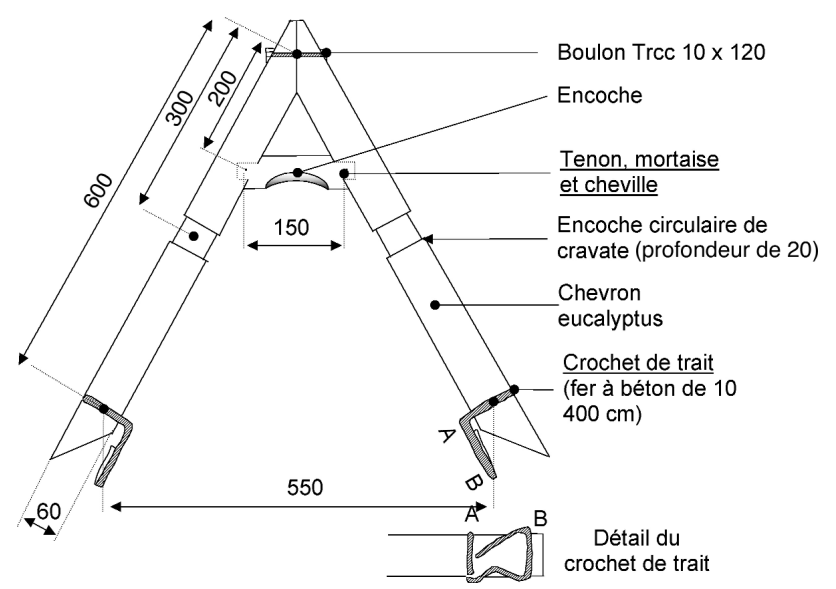

Figure 3 : jouguet monobovin Irad BT (en souligné, les modifications suggérées par les agriculteurs).

\section{Encadré 1}

Principes d'utilisation du jouguet

\section{INSTALLATION DU JOUGUET}

L'installation du jouguet se déroule en trois étapes.

1. Poser le jouguet sur la bosse de l'animal.

2. Attacher la cravate au niveau du cou de l'animal sans trop la serrer (lorsque l'on tend les traits, le jouguet doit se placer en position oblique suivant la ligne de traction, sans étrangler le bovin).

3. Accrocher le palonnier à l'équipement agricole. Pour améliorer le réglage du talonnage de I'outil agricole (charrue), il est possible de rajouter une corde de quelques décimètres entre le crochet du palonnier et celui de l'outil.

\section{DRESSAGE DU BOVIN}

Le dressage est une opération très importante. Cependant, lors des nombreuses démonstrations effectuées avec les paysans, il a été constaté que dans la très grande majorité des cas, les bovins habitués à travailler par paires s'habituaient sans difficulté et presque immédiatement au jouguet monobovin.

Avec un animal non-dressé, il s'agit de l'amener progressivement à travailler seul. Pour l'accoutumer au harnais, on commence par placer le jouguet sur la bosse à l'arrêt. Puis, petit à petit, on habitue l'animal à marcher avec le jouguet en lui faisant tracter une charge d'abord légère, puis progressivement de plus en plus lourde. L'opération dure au minimum une semaine. Ensuite, en douceur, le travail peut être effectué en plein champs pour des travaux de sarclage et de buttage. Après une saison de culture, le bovin est apte à travailler avec le jouguet.

\section{RECOMMANDATIONS}

1. Il faut éviter de trop serrer la cravate car on risque d'entraver la respiration et la circulation du sang du bœuf.

2. Après le travail, il est préférable de suspendre le jouguet afin de le protéger des vermines (termites et autres).

3. Un bovin seul se fatigue plus rapidement qu'une paire ; il y a donc lieu d'accorder des repos fréquents à l'animal durant le travail et d'éviter les labours trop profonds.

\section{Jouguet Irad BF}

Ce jouguet (figure 4) comprend une pièce de bois taillée dans un madrier (7 à $8 \mathrm{~cm}$ x $20 \mathrm{~cm}$ de section). En forme de demi-cercle, elle épouse parfaitement la forme de la bosse. Cette pièce de bois est entaillée extérieurement sur la tranche par une gorge de $2 \mathrm{~cm}$ où passe un fer à béton de 12 (d'où son nom, $\mathrm{BF}=$ bois fer). Le fer à béton $(160 \mathrm{~cm})$ comprend une partie en demi-cercle et deux bras de $30 \mathrm{~cm}$ environ qui se terminent par les crochets des traits. Le fer est plié à froid. Les auteurs ont ajouté une dossière de $75 \mathrm{~cm}$ (angle trait-dossière $=70^{\circ}$ ) afin d'améliorer la stabilité du jouguet. Elle se raccorde aux crochets des bras du jouguet. Les brins de la cravate sont plus courts (50 et $90 \mathrm{~cm}$ de long) que ceux du modèle Irad BT et se fixent au niveau des bras. Ce modèle est plus léger que le précédent et plus facile à réaliser. Toutefois, il requiert l'emploi d'une machine à bois.

* Une version en chevron de menuiserie industrielle a été réalisée mais finalement abandonnée en raison d'un coût prohibitif 


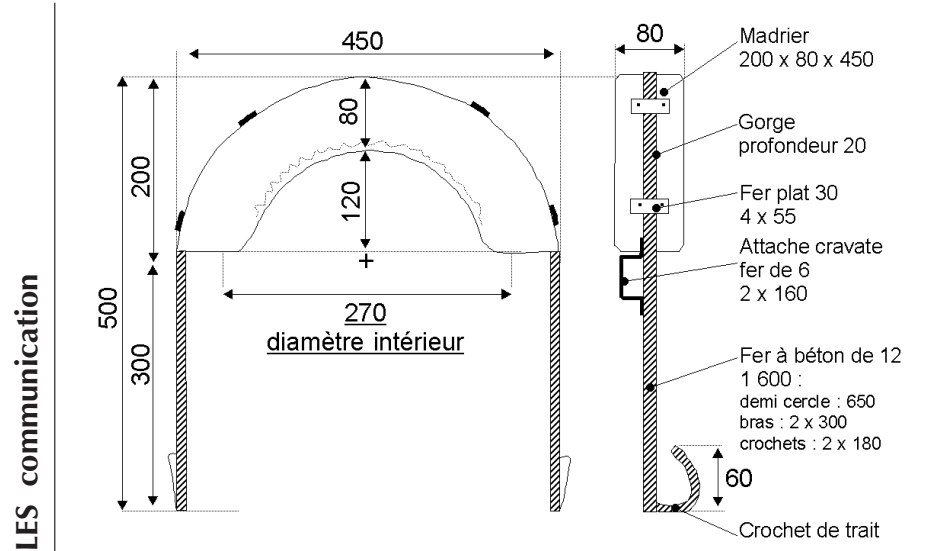

Figure 4 : jouguet monobovin Irad BF (en souligné, les modifications suggérées par les agriculteurs).

\section{Sanglage et palonnier}

Les sanglages ont été réalisés au moyen d'une sangle en Nylon de très haute résistance $(250 \mathrm{kN})$ importée par la Sodécoton $(560 \mathrm{Fcfa} / \mathrm{m})$. La longueur des traits est de $160 \mathrm{~cm}$ (figure 2). La bande de Nylon importée peut éventuellement être remplacée par la sangle de Nylon que l'on trouve couramment sur les marchés locaux. Cependant, il convient de la doubler pour augmenter la résistance. Les traits se raccordent à un palonnier (bûche d'eucalyptus de $65 \mathrm{~cm} ; 6 \mathrm{~cm}$ de diamètre) par deux crochets de forme triangulaire (fer à béton de 10) (le méplat du crochet permet de mieux répartir la traction sur la couture de la bande de Nylon). Le crochet d'attelage est circulaire (fer à béton de 10). Les trois crochets sont fixés par enroulement à froid autour de la pièce de bois afin d'éviter un perçage qui l'affaiblirait.

\section{Enquête d'acceptabilité}

Les deux modèles ont été utilisés par tous les paysans volontaires pour le test et par un nombre encore plus important de voisins venus emprunter le matériel. Par ordre décroissant d'importance, les jouguets ont servi pour le sarclage, le labour et le buttage. La préférence des agriculteurs s'est très nettement portée sur le modèle Irad BF qui, selon leur propre expression, habille bien l'animal.

Le problème de stabilité dans les tournières a été totalement résolu avec le modèle Irad BF (100 p. 100 de satisfaction). En revanche, certains utilisateurs du modèle Irad BT (35 p. 100) se sont plaints de son instabilité sur des petits bœufs et l'ont expliqué par l'angle des montants trop ouvert.

Les paysans ont jugé le matériel suffisamment solide (un seul cas de casse sur le crochet palonnier-outil). Pour le modèle Irad BF, aucune modification n'a été demandée pour augmenter sa solidité. Concernant le modèle Irad BT, une majorité d'agriculteurs ont souhaité que les lanières de caoutchouc qui maintiennent la pièce centrale soient remplacées par des chevilles et que l'encoche de fixation des traits soit remplacée par un crochet en fer à béton de 10 de manière à éviter la torsion de la sangle qui pourrait accélérer son usure.

Malgré la taille unique proposée pour chaque modèle, les paysans estiment que les deux jouguets sont confortables pour l'animal (peu de signes d'énervement ou de douleur, pratiquement pas de blessures et une accoutumance au jouguet n'excédant pas un jour). Les paysans ont parfois remarqué des signes d'énervement au début (refus, mouvements de tête, fuite de l'effort). Cependant, aucun refus définitif n'a été signalé. Selon les déclarations des agriculteurs, la vitesse de travail d'un monobovin est comparable à celle d'une paire de bovins Avec le modèle Irad BT, il est arrivé parfois lors des premiers essais que la pointe du triangle frappe la nuque du bovin. Mais avec l'habi- tude, l'animal apprend à marcher en baissant légèrement la tête et le défaut disparaît. La taille unique était une demande du commanditaire en vue de simplifier la fabrication du matériel en série. Quelques problèmes d'essayage se sont posés avec des animaux « hors gabarit ». Lorsqu'il s'agit de petits bœufs, les auteurs suggèrent d'enrouler un tissu sur la partie centrale du jouguet afin d'améliorer le contact bosse-jouguet. Si en revanche la bosse de l'animal est trop importante (bovins de grande taille, zébus Goudali), il est possible d'élargir l'arrondi de l'Irad BF en rabotant les angles (il n'existe pas de solution pour le modèle Irad BT).

Quelques paysans se sont plaints de la casse des cultures par le palonnier. Après vérification, il s'est avéré que ces personnes n'avaient pas respecté l'interligne de $80 \mathrm{~cm}$. Enfin, des paysans ont suggéré un système de réglage de la longueur des traits pour jouer sur le réglage du talonnage de l'outil. Techniquement et économiquement, il est préférable de conserver des traits fixes, plus précisément pas trop longs pour éviter que l'animal ne s'y prenne les membres arrière dans les virages, et d'ajouter simplement une chaînette ou une corde entre le palonnier et l'outil.

\section{Test des jouguets à Mafa Kilda}

Les performances comparées des attelages mesurées avec les deux modèles de jouguets sont indiquées dans le tableau I. Les temps de travaux et les vitesses sont des moyennes arithmétiques calculées sur trois attelages différents, observés durant leur travail pendant deux heures environ. Les temps de travaux (h/ha) ont été calculés à partir des relevés de vitesse moyenne (la vitesse moyenne tient compte des temps d'arrêts) et d'écartement entre deux sillons consécutifs. Les vitesses sont des valeurs instantanées ne tenant pas compte des arrêts.

Le harnais monobovin permet de réaliser tous les travaux de culture attelée, labour, sarclage et buttage avec cependant des différences entre les performances des animaux pour ces différentes opérations. Pour les travaux de sarclage et de buttage, les résultats ont montré que le bovin de trait a été plus performant avec un jouguet Irad BF ; le temps de travail a été plus court et la vitesse plus élevée ( 6 contre $7,5 \mathrm{~h} / \mathrm{ha}$ et 5 contre $4 \mathrm{~km} / \mathrm{h}$ ). La contre-performance du modèle Irad BT s'explique surtout par les pertes de temps dans les virages à replacer le jouguet.

$\mathrm{Au}$ labour, on constate que les temps de travaux ont été éminemment variables selon les cultures ( $15 \mathrm{~h} / \mathrm{ha}$ pour le coton contre $30 \mathrm{~h} / \mathrm{ha}$ pour le maïs), pour des vitesses instantanées comparables (2,5 à

\section{Tableau I}

Performances comparées des attelages monobovins Irad BF et Irad BT sur coton et maïs à Mafa Kilda *

\begin{tabular}{|c|c|c|c|c|c|}
\hline \multirow[t]{2}{*}{ Opérations } & \multirow[t]{2}{*}{ Cultures } & \multicolumn{2}{|c|}{$\begin{array}{l}\text { Temps de travail } \\
\text { (h/ha) }\end{array}$} & \multicolumn{2}{|c|}{$\begin{array}{l}\text { Vitesse } \\
(\mathrm{km} / \mathrm{h})\end{array}$} \\
\hline & & Irad BF & Irad BT & Irad BF & Irad BT \\
\hline \multirow[t]{2}{*}{ Labour } & Coton & 12 & 16 & 3,0 & 2,8 \\
\hline & Maïs & 33 & 28 & 3,1 & 2,3 \\
\hline \multirow[t]{2}{*}{ Sarclage } & Coton & 6 & 8 & 5,1 & 3,8 \\
\hline & Maïs & 6 & 7 & 5,3 & 3,6 \\
\hline \multirow[t]{2}{*}{ Buttage } & Coton & 6 & 7 & 5,9 & 2,5 \\
\hline & Maïs & 5 & 7 & 5,3 & 3,2 \\
\hline
\end{tabular}

* Source : Kpoumié E., 1998, Mémoire diplôme Ingénieur agronome, Fasa, Dschang, Cameroun 
$3 \mathrm{~km} / \mathrm{h}$ ). Un tel écart a conduit les auteurs à formuler l'hypothèse que les écartements entre sillons consécutifs diffèrent selon les cultures. Malheureusement, cette hypothèse n'a pas été vérifiée et les normes paysannes en la matière restent inconnues. Cependant, Vandendael (11) a montré que pour la culture du coton les agriculteurs laissent des bandes de terre non travaillées entre les sillons (il a mesuré un écartement moyen entre deux sillons de $45 \mathrm{~cm}$ pour une largeur de sillon de $25 \mathrm{~cm}$ ). Le coton, avec son enracinement pivotant, valorise bien ce type de pratique culturale économique en temps de travail si l'on sème sur la partie travaillée. Pour la culture du maïs, les agriculteurs resserrent probablement les sillons pour optimiser l'enracinement peu pénétrant de cette culture.

En 1999, 2000 et 2001, les auteurs ont mis à la disposition du groupement de producteurs de coton de Mafa Kilda plusieurs exemplaires de jouguet Irad BF. Les agriculteurs se sont organisés pour utiliser le matériel à tour de rôle. Ils se sont bien approprié la technique pour le labour et les opérations d'entretien. Les nombreux agriculteurs du village propriétaires d'un seul bovin de trait, 11 p. 100 selon Cuvier (1), ont ainsi trouvé ainsi une alternative à la pratique d'association, peu flexible et bien souvent contraignante et peu satisfaisante pour le respect des dates d'intervention.

\section{Prix de revient des jouguets}

Le modèle Irad BF est le moins cher (tableau II). Il coûte 7500 Fcfa contre 8700 Fcfa pour le modèle Irad BT (tableau III). La main-d'œuvre représente respectivement 30 et 44 p. 100 du prix de revient des modèles Irad BF et Irad BT. Pour le modèle Irad BT, c'est le travail du menuisier qui coûte cher. Il faut également noter que la bande de Nylon représente une part considérable du prix de revient des jouguets, environ $3300 \mathrm{Fcfa}$ (soit 37 à 44 p. 100 du prix de revient). Cependant, ce choix technique n'a pas été remis en cause par les paysans. Ils apprécient particulièrement la solidité de ce matériau et estiment cette dépense justifiée.

Environ 70 p. 100 des paysans interrogés jugent que le prix du modèle Irad BF est correct contre seulement 35 p. 100 pour le modèle Irad BT. Environ 25 p. 100 des paysans se déclarent prêts à acheter le jouguet au comptant. Les autres suggèrent une acquisition au moyen d'un « crédit de campagne » proposé par la Sodécoton.

\section{Tableau II}

Prix de revient du jouguet Irad BF

$\begin{array}{lrr} & \text { Fcfa } & \text { \% du total } \\ & & \\ \text { Jouguet } & & \\ \text { Madrier }(45 \mathrm{~cm} \times 20 \mathrm{~cm} \times 7 \text { à } 8 \mathrm{~cm}) & 600 & 8 \\ \text { Fer de } 12(160 \mathrm{~cm}) & 600 & 8 \\ \text { Fer plat } 3 \mathrm{~cm}(4 \times 5,5 \mathrm{~cm}) & 100 & 1 \\ \text { Pointes de } 6(8) & 20 & 0 \\ \text { Fer de } 6(2 \times 16 \mathrm{~cm}) & 50 & 1 \\ \text { Cravate } & & \\ \text { Bande de Nylon }(50 \mathrm{~cm}+90 \mathrm{~cm}) & 780 & 10 \\ \text { Fer de } 6(2 \times 19 \mathrm{~cm}) & 60 & 1 \\ \text { Traits et palonnier } & & \\ \text { Bande de Nylon }(2 \times 190 \mathrm{~cm}+75 \mathrm{~cm}) & 2550 & 34 \\ \text { Fer de } 10(3 \times 40 \mathrm{~cm}) & 320 & 4 \\ \text { Eucalyptus }(65 \mathrm{~cm} ; \mathrm{d}=6 \mathrm{~cm}) & 120 & 2 \\ \text { Sous-total matériel } & 5200 & 70 \\ \text { Sous-total main d'œuvre } & 2270 & 30 \\ \text { Total } & 7470 & 100\end{array}$

\section{DISCUSSION ET CONCLUSION}

Si l'on se réfère au cahier des charges, on constate que les deux modèles sont faisables avec des matériaux et une technologie disponibles localement, excepté cependant pour un élément important, la bande de Nylon, au demeurant très appréciée par les paysans. Le prix de revient des deux modèles est inférieur à 10000 Fcfa, mais il est plus avantageux pour l'Irad BF (7 500 Fcfa environ). A la suite des multiples tests de longue durée au champ, l'absence d'avarie permet de conclure que les jouguets sont suffisamment solides si l'on apporte des transformations mineures. Ils permettent de passer entre les lignes de culture sans provoquer de casse si l'on respecte un interligne de $80 \mathrm{~cm}$. Le problème de renversement dans les tournières, rencontré par la Sodécoton en 1997, a été totalement résolu avec le jouguet Irad BF. Cependant, ce problème a persisté dans 35 p. 100 des cas avec le modèle Irad BT, notamment sur les petits bovins. Malgré une taille unique, les deux modèles s'adaptent à l'encolure de la grande majorité des zébus, excepté pour les individus d'un gabarit très largement supérieur à la moyenne.

Les deux jouguets ont été bien appréciés avec cependant une très nette préférence pour le modèle Irad BF. Finalement, les modifications proposées par les agriculteurs n'ont pas été très nombreuses. Les figures 3 et 4 prennent en compte les modifications suggérées par les agriculteurs au cours de l'étape de prévulgarisation, c'est-àdire, brièvement, l'augmentation du diamètre intérieur du jouguet Irad $\mathrm{BF}$, le remplacement des encoches de fixation des traits des crochets sur le modèle Irad BT et le remplacement des lanières de caoutchouc fixant la pièce centrale du modèle Irad BT par deux chevilles qui augmentent ainsi la solidité du montage. Enfin pour les deux modèles, les auteurs suggèrent d'ajouter une corde $(1 \mathrm{~m})$ au crochet d'attelage pour affiner le réglage du talonnage de la charrue.

A l'issue de cette phase d'essai, la Sodécoton a opté pour le développement du modèle Irad BF, manifestement plébiscité par les agriculteurs. Elle fabrique les jouguets dans ses propres ateliers et les cède au comptant, sans bénéfice, au prix de 7000 Fcfa. Quatre années après la phase de prévulgarisation, elle a vendu 620 exemplaires (7). L'essentiel des acquisitions ont eu lieu dans la région

\section{Tableau III}

Prix de revient du jouguet Irad BT

\begin{tabular}{|c|c|c|}
\hline & Fcfa & $\%$ du total \\
\hline \multicolumn{3}{|l|}{ Jouguet } \\
\hline Eucalyptus $(2 \times 80 \mathrm{~cm} ; d=6$ à $8 \mathrm{~cm})$ & 320 & 4 \\
\hline Eucalyptus $(25 \mathrm{~cm} ; \mathrm{d}=9 \mathrm{~cm})$ & 50 & 1 \\
\hline Boulon Trcc $10 \times 120+$ rondelle & 490 & 6 \\
\hline Lanière caoutchouc (2 x 100 cm) & 150 & 2 \\
\hline Fer de $10(2 \times 40 \mathrm{~cm})$ & 200 & 2 \\
\hline \multicolumn{3}{|l|}{ Cravate } \\
\hline Bande de Nylon $(70 \mathrm{~cm}+110 \mathrm{~cm})$ & 1010 & 12 \\
\hline Fer de $6(2 \times 19 \mathrm{~cm})$ & 60 & 1 \\
\hline \multicolumn{3}{|l|}{ Traits et cravate } \\
\hline Bande de Nylon (2 x 190 cm) & 2130 & 25 \\
\hline Fer de $10(3 \times 40 \mathrm{~cm})$ & 320 & 4 \\
\hline Eucalyptus $(65 \mathrm{~cm} ; \mathrm{d}=6 \mathrm{~cm})$ & 120 & 1 \\
\hline Sous-total matériel & 4850 & 56 \\
\hline Sous-total main d'œuvre & 3810 & 44 \\
\hline Total & 8660 & 100 \\
\hline
\end{tabular}


de Touboro (45 p. 100), puis dans les autres régions de la province du Nord (40 p. 100 au total). Le développement de la technique commence tout juste dans les régions de l'extrême nord du Cameroun. Aujourd'hui, la collaboration se poursuit avec un nouveau partenaire, Manucycle (Pme camerounaise), pour la mise au point d'une charrette monobovine et d'un harnais basé sur le jouguet Irad BF (figure 5).
Ailleurs en Afrique sub-saharienne, les bovins de trait sont généralement attelés par paires, alors que les ânes, animaux plus légers, sont utilisés seuls. La traction monobovine se présente comme une technologie intermédiaire en termes de capacité de traction et de coût d'investissement. Ce type d'innovation a donc une pertinence qui dépasse les frontières du Nord Cameroun et mériterait d'être testé dans d'autres pays.

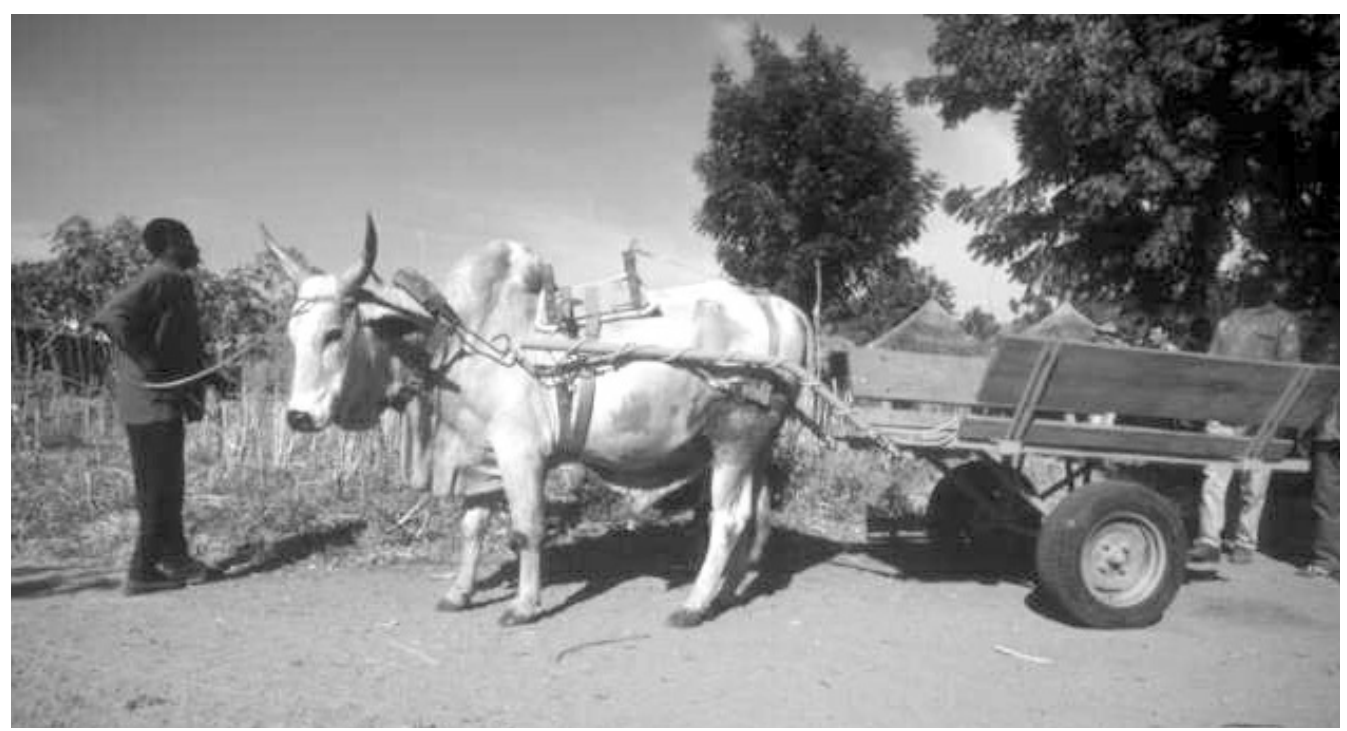

Figure 5 : charrette monobovine en cours d'expérimentation (cliché : B. Faye, Cirad-emvt).

\section{BIBLIOGRAPHIE}

1. CUVIER L., 1999. Etude des pratiques et des stratégies paysannes de traction animale dans la zone cotonnière du Nord Cameroun : cas du terroir de Mafa-Kilda. Mémoire Dess Productions animales en régions chaudes, Cirad-emvt, Montpellier, France, 82 p.

2. GRET, 1984. Le point sur les harnais pour la traction animale. Paris, France, Gret, Grdr, 50 p. (Coll. Le point sur, $n^{\circ} 5$ )

3. KPOUMIE E., 1998. Conception et expérimentation de deux types de jouguets en milieu contrôlé et en milieu paysan au Nord Cameroun. Mémoire diplôme Ingénieur agronome, option Productions animales, Fasa, Université de Dschang, Cameroun, 50 p.

4. LE THIEC G., 1994. Etude sur le travail animal et I'optimisation de I'efficacité du couple attelage-outil. Mission d'appui : améliorations des harnachements. Montpellier, France, Cirad-sar, 10 p. (Rapport de mission 67/94)

5. LE THIEC G., Ed. sci., 1996. Agriculture africaine et traction animale. Montpellier, France, Cirad, 355 p. (Coll. Technique)

6. NJOYA A., BOUCHEL D., NGO TAMA A.C., 1995. Détermination indirecte du poids des bovins par la barymétrie en zone soudano- sahélienne du Cameroun. Fiche technique, Projet Garoua 2. Garoua, Cameroun, Ira/Irzv/Cirad, 2 p.

7. SODECOTON, 2001. Rapport semestriel de mai à octobre 2001 Campagne agricole 01/02. Garoua, Cameroun, Dpa Sodécoton, 56 p.

8. STARKEY P., 1994. Systèmes d'attelage et matériels à traction animale. Eschborn, Allemagne, Gate, Gtz Gmbh, 278 p.

9. VALL E., 1996. Capacités de travail, comportement à l'effort et réponses physiologiques du zébu, de l'âne et du cheval au Nord Cameroun. Thèse Doct., Ensam, Montpellier, France, 418 p.

10. VALL E., OUMAROU A., KPOUMIE E., 1998. Culture attelée : équipements. Jouguets monobovins. Document de travail. Garoua, Cameroun, Irad, $17 \mathrm{p}$.

11. VANDENDAEL L., 2000. Les paysans du Nord Cameroun et leurs charrues : un outil, son utilisation et sa fabrication. Mémoire Diplôme Ingénieur agronome, orientation Génie rural, FusaGx, Gembloux, Belgique, $84 \mathrm{p}$.

Reçu le 14.01.2002, accepté le 28.06.2002 


\section{Summary}

Vall E., Abakar O., Kpoumié E. Developing Single-Ox Yokes for Draft Animal Activities in the Savannah Zone of North Cameroon

In North Cameroon, farmers use a pair of oxen or a donkey for plowing and crop maintenance work. Before 1997, the use of a single ox for draft animal cultivation was not taken into account by development projects. On the other hand, maneuvering a pair of oxen is difficult in the spacing of cultivated fields (plants trampled, stalks broken by animals and traction chains, difficult maneuvering at the end of rows). The farmers did not adopt the straddling yoke because its large size $(160 \mathrm{~cm})$ makes maneuvering difficult at the end of rows. Single-ox drafting can solve these difficulties. In 1998, at the request of the Society for Cotton Development in Cameroon (SODECOTON), the Institute for Agronomic Research and Development (IRAD) developed and tested under farmers' conditions two models of single-ox yokes. The IRAD BF model is characterized by a semicircular piece of wood that closely fits the animal bump (cost: 7500 FCFA). The IRAD BT model is made of three pieces of wood assembled into a triangle (cost: 8700 FCFA). Volunteer farmers tested a series of 50 samples of each model. The majority preferred the IRAD BF model because of its unfailing stability and its better fit on the animal bump. The weak points of the IRAD BT model were the following: high cost, defective assembly of the hames, unsteadiness when turning. Since 1999, SODECOTON has sold 620 samples of the IRAD BF model. The development of a single-ox cart is currently under study.

Key words: Cattle - Harness - Draft animal cultivation Animal power - Cameroon.

\section{Resumen}

Vall E., Abakar O., Kpoumié E. Desarrollo de pequeños yugos mono bovinos para el cultivo con atelaje en la zona de sabana del norte de Camerún

Los agricultores del norte de Camerún utilizan un par de bueyes o bien un asno para labrar y para los trabajos de mantenimiento de los cultivos. Antes de 1997, el uso de un "mono buey" para el cultivo con atelaje no era tomado en cuenta para el desarrollo. Sin embargo, en una parcela cultivada, el paso de un par de bovinos por la línea media presenta dificultades para el conductor (pisoteo de las plantas, tallos quebrados por la cadena de tracción, dificultades de maniobra hacia el final de las filas). El yugo de pierna no fue adoptado por los agricultores, debido a que su envergadura $(160 \mathrm{~cm})$, dificulta la maniobra del atelaje hacia el final de las filas. El atelaje mono bovino soluciona estas dificultades. En 1998, por pedido de la Sociedad de desarrollo de algodón en Camerún (Sodecoton), el Instituto de investigación agrícola para el desarrollo (Irad) realizó y probó en medio campesino, dos modelos de pequeños yugos mono bovinos. El modelo Irad BF se caracteriza por una pieza de madera en forma de medio círculo, que se encaja sobre la giba de un cebú (7500 Fcfa). El modelo Irad BT está compuesto de tres piezas de madera ajustadas en triangulo (8700 Fcfa). Una serie de 50 ejemplares de cada modelo fueron probados por agricultores voluntarios. Una mayoría manifestó preferencia por el modelo Irad BF, debido a que es perfectamente estable y mejor ajustado sobre la giba del animal. Los puntos débiles del modelo Irad BT son los siguientes: precio demasiado elevado, defecto de montaje de los montantes, inestabilidad en las curvas. Desde 1999, la Sodecoton vendió 620 ejemplares del modelo Irad BF. El trabajo sigue, con el diseño de una carreta mono bovina.

Palabras clave: Ganado bovino - Arnes - Cultivo a tracción animal - Energía animal - Camerún. 